\title{
An Ultrafast Maximum Power Point Setting Scheme for Photovoltaic Arrays Using Model Parameter Identification
}

\author{
Zhaohui Cen \\ Qatar Environment and Energy Research Institute (QEERI), Member of Qatar Foundation, P.O. Box 5825, Doha, Qatar \\ Correspondence should be addressed to Zhaohui Cen; cenzhaohui@gmail.com
}

Received 3 December 2014; Revised 1 March 2015; Accepted 24 March 2015

Academic Editor: Francisco A. S. Neves

Copyright (C) 2015 Zhaohui Cen. This is an open access article distributed under the Creative Commons Attribution License, which permits unrestricted use, distribution, and reproduction in any medium, provided the original work is properly cited.

\begin{abstract}
Maximum power point tracking (MPPT) for photovoltaic (PV) arrays is essential to optimize conversion efficiency under variable and nonuniform irradiance conditions. Unfortunately, conventional MPPT algorithms such as perturb and observe (P\&O), incremental conductance, and current sweep method need to iterate command current or voltage and frequently operate power converters with associated losses. Under partial overcast conditions, tracking the real MPP in multipeak $P-I$ or $P$-V curve model becomes highly challenging, with associated increase in search time and converter operation, leading to unnecessary power being lost in the MPP tracking process. In this paper, the noted drawbacks in MPPT-controlled converters are addressed. In order to separate the search algorithms from converter operation, a model parameter identification approach is presented to estimate insolation conditions of each PV panel and build a real-time overall $P-I$ curve of PV arrays. Subsequently a simple but effective global MPPT algorithm is proposed to track the MPP in the overall P-I curve obtained from the identified PV array model, ensuring that the converter works at the MPP. The novel MPPT is ultrafast, resulting in conserved power in the tracking process. Finally, simulations in different scenarios are executed to validate the novel scheme's effectiveness and advantages.
\end{abstract}

\section{Introduction}

Globally, installed photovoltaic (PV) capacity has passed $100 \mathrm{GW}$ while the US is projected to install $24 \mathrm{GW}$ by 2015 [1-3]. However, the low-energy conversion efficiency of PV materials remains a barrier to the prolific growth of solar electricity and requires maximum power point (MPP) of the arrays to maximize conversion efficiency [4-6]. Due to timevarying operating conditions such as insolation, temperature, and atmospheric particulates, the PV panels or arrays need effective and fast MPP tracking (MPPT) algorithms or converters to attain the best harvesting efficiency [7-10].

Many MPPT algorithms have been proposed to achieve best energy harvesting efficiency under different operating conditions. Several well-known MPPT algorithms, such as hill climbing, perturb and observe (P\&O), incremental conductance, ripple correlation control, and $\mathrm{d} p / \mathrm{d} v$ or $\mathrm{d} p / \mathrm{d} i$ feedback, can search the MPP in an efficient and timely way $[5,9,11-13]$. However, these algorithms cannot track the MPP under nonuniform insolation. Addressing nonuniform insolation problems such as partial shading $[5,14,15]$, some improved global MPPT algorithms have been developed to avoid tracking the nonmaximum peak points in the nonnormal global $P-V$ or $P-I$ curves $[1,16-20]$. Typically, the basis of all these GMPPT algorithms increases the complexity of the search algorithms. Theoretically, MPP convergence can be achieved by many search algorithms if there is no time-related penalty, which is associated with algorithm run frequency, step length, algorithmic complexity, and convergence time related to the characteristics of the $P-V$ or $P-I$ curve.

Whichever MPPT algorithm is utilized, two distinct actions are engaged in the MPP search process. The first action is to calculate the next-step command current or voltage at the current step; the second action is to set this current or voltage commanding at the DC-DC converter. In all the algorithms considered above, the two actions are linked to execute together for each searching step. Therefore, the total execution time of the current or voltage switching operation is dominated by and depends on the total execution time of the search algorithms during numerous iterations. 
Consequently, considerable time and energy are unnecessarily lost in the search while the MPP is directly available in real-time but idling pending search update. Also, the MPP of PV panels depends on the $P-I$ or $P-V$ curve, which themselves depend on the ambient conditions such as insolation and temperature, so the MPP should be theoretically employed analyzing the real-time ambient condition and inferring from the mathematical relationship between the ambient condition parameters and the $P-V$ or $P-I$ curve model.

To address the issues discussed above, a novel ultrafast maximum power point setting scheme based on model parameter identification is proposed. The scheme addresses the real-time availability of MPP and also the drawback in the conventional MPPT algorithms for PV arrays under both uniform and nonuniform insolation. In this scheme, an analytical model parameter identification approach is presented to identify the insolation parameter for each PV panel using their measured voltage and current. Based on real-time estimated insolation parameters for each solar panel, an overall $P-I$ or $P-V$ curve model is built in the controller and the MPP is rapidly calculated by an MPP search algorithm. Subsequently, the computed current or voltage at the MPP is sent to command the converter to work at the MPP directly.

The main contribution and difference between the proposed MPPT scheme and other MPPT algorithms is that the proposed MPPT algorithm is executed in the controller for virtually constructed $P-I$ or $P-V$ curve derived from the identified insolation parameters, while other MPPT algorithms are executed for real $P-I$ or $P-V$ curves measured during the search process. Because the proposed MPPT algorithm is based on the virtual $P-I$ or $P-V$ curve in the controller, MPP search alone is executed in the controller and can be separated from the converter operation. Theoretically, that means only one-time current or voltage setting is needed to make the converter work in the MPP once the virtual identified $P$ $I$ curve correlates with the real $P-I$ curve. Therefore, the proposed novel MPPT approach is ultrafast in cases where some prior knowledge about PV panel characteristics is available. Although it needs some predefined parameters to build the PV numerical model in advance and loses some flexibility which can be addressed by other approaches, it is acceptable for engineering implementation and a trade-off optimization between time-consumption and flexibility. Also it has another benefit that the insolation condition for each piece of PV panel can be estimated rather than measured by additional hardware insolation sensors. Compared with our former work in [19], this paper addresses the unsolved timeconsumption problem in the global MPPT by a novel model identification approach.

The remainder of this paper is organized as follows. In Section 2, the drawbacks of conventional MPPT algorithms, low-efficiency searching and power-loss problems, are presented and discussed. The ultrafast MPP searching scheme is introduced in Section 3. In Section 4, we present the simulation results under both uniform and nonuniform insolation conditions where the proposed MPPT scheme is applied to PV energy harvesting based on typical DC-DC converters. Finally, conclusion is provided in Section 5.

\section{Problem Formulation}

2.1. Drawback of Current MPPT Methods. A typical MPPT based PV DC-DC power conversion circuit and P\&O MPPT algorithm diagram are shown in Figure 1. From the diagram we can see that the MPPT controller has interactions with the DC-DC converter in a closed loop with inputs of measured current and voltage and output of the current or voltage command. It also depicts that the operation on voltage increasing or decreasing depends on the P\&O algorithm's calculation and comparison in each searching step. As can be discovered from the algorithm procedure, after it commands the converter voltage, it cannot iterate to next-step search until the converter output power value subject to the set current or voltage is fed back to the algorithm and a new search point is calculated. Also, the power converter's operation takes a longer time than the MPPT controller calculation in each step while the MPPT controller waits to measure the output power of the converter. The drawback of conventional MPPT methods is that the majority of execution time is spent on the converter operation but not on algorithm calculation.

\subsection{Low-Efficiency Search Caused Power Loss and Potential} Reliability Problems. Converter operation is engaged in the iterative search process, resulting in power being unnecessarily lost in this procedure. Furthermore, variations of voltage or current are discontinuous and the associated steps may be very large. Consequently, these discontinuous and largestep current or voltage switching actions will reduce the mean time to failure of components such as IGBT or MOSFET due to increased stress, with associated switching losses, and increased ripple current in the converter circuits.

\subsection{Feasibility of Ultrafast Search and Availability of MPP.} Assuming that the $P-V$ curve of solar panel is available in real-time, the multiple interaction for identifying the MPP between the MPPT controller and the DC-DC power converter is unnecessary. Once the $P-V$ curve is available, it is simple to compute the MPP numerically without interactions with the DC-DC converter, and the DC-DC converter can be set in MPP directly because the MPP tracking process has been already set in the controller in advance. The key problem here is to obtain an overall $P-V$ curve available in real-time. As stated before, the $P-I$ or $P-V$ curve model depends on the ambient conditions such as insolation and temperature. So, the key ambient condition factors such as insolation can be theoretically identified from some sample data consisting of PV panel's voltage and current and the known general numerical model of the PV cell, which is generally fixed for a predefined PV module of a specific manufacturer in a known array configuration. In order to obtain this model, some parameters such as $A$ (diode ideality factor), $R_{s}$ and $R_{p}$ (series and parallel resistances), and the array configuration used (all series, all parallel, and series-parallel) may be predefined in advance. Although it will lose some flexibility for this model, it is acceptable for engineering because this information is fixed in operation and easy to obtain in advance. Once the real-time insolation for each PV panel is available, the overall $P-I$ or $P-V$ curve model for PV arrays can be calculated 


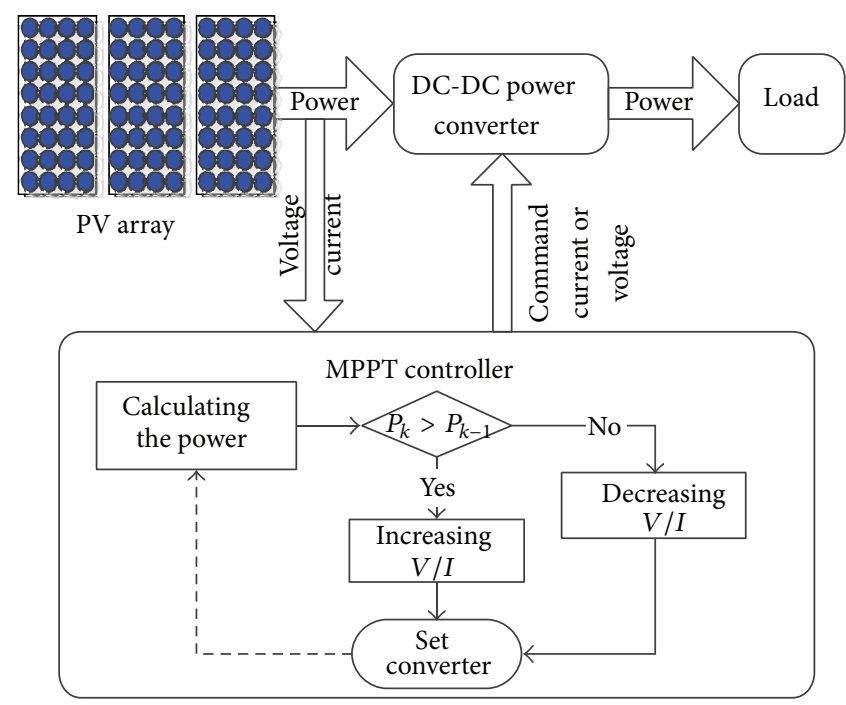

Figure 1: P\&O MPPT DC-DC power conversion.

virtually, and then the MPP is available from the search based on the virtual $P-I$ or $P-V$ curve.

2.4. PV Model Identification Parameters Selection. Generally, we can classify the PV panel parameters into two sets. One set is temperature and irradiance; the other is $A, R_{s}$ and $R_{p}$, and the array configuration. The former parameter set depends on the ambient conditions and it varies faster, which requires parameter identification and tracking in real-time, forming the main challenge. The later set depends on the PV's inherit characteristics and its installation configuration and it is generally fixed and stable in operation. Due to a tradeoff between simplicity of online identification algorithm and parameter's priorities, the first set is considered as inputs in the studied PV model. Since temperature changes slower than irradiance and it can be measured by thermocouple sensor, the temperature is assumed to be a known constant in this study.

\section{Ultrafast MPP Setting Scheme}

In this section, the ultrafast MPP setting scheme is presented in two parts. Firstly, the insolation parameter identification based on algebraic equations solving is proposed. Secondly, the real-time $P-I$ curve generation is presented. Finally, the converter operation with the MPP is described. An overall diagram about the ultrafast MPP setting scheme is shown in Figure 2. The MPPT controller with ultrafast MPP setting scheme has almost the same hardware configuration with the conventional MPPT controller, but the algorithm scheme inside is completely novel. The interaction between the MPPT controller and the DC-DC power converter is only executed twice during one-time MPPT tracking period (generally from several minutes to half an hour [21-24]); the first instance is to measure a sample data set of current and voltage from solar panel to the converter. The second instance is to command the converter with calculated current or voltage at the MPP. The "one-time on-off control" in Figure 2 means that one-time interaction with DC-DC converter (turn-on and turn-off) is needed for initial measurement. Between the two interactions, the insolation identification, the $P-I$ curve model building, and the MPP tracking based on the $P-I$ curve are executed in the controller at an ultrafast speed. In order to simplify the nonuniform insolation problem, hereby a hypothesis is presented for convenience.

Hypothesis 1. If a PV panel/cell unit experiences partial shading, dust, water, aging, or any adverse conditions, the $P$ $V$ or $P-I$ curve model equation will still hold or can be seen as a one-peak shape.

Comment 1. Because PV panel size is smaller than PV arrays, the noninsolation has no or weak effect at one PV panel unit although PV arrays will have a multipeak $P-V$ or $P-I$ curve model. So it is feasible and acceptable to make this hypothesis for a PV panel unit.

3.1. Insolation Parameter Identification. As previously stated, the general numerical model of a PV cell can be depicted in Figure 3.

The circuit mathematical model can be denoted as below:

$$
\begin{gathered}
I_{\mathrm{sc}}-I_{D}-\frac{V_{D}}{R_{p}}-I_{\mathrm{PV}}=0, \\
I_{D}=I_{o}\left(\mathrm{e}^{V_{D} / V_{T}}-1\right), \\
V_{\mathrm{PV} \text { cell }}=V_{D}-R_{s} I_{\mathrm{PV}},
\end{gathered}
$$

where $I_{D}$ and $V_{D}$ denote the diode current and voltage and $V_{T}$ is the diode thermal voltage. $I_{\mathrm{PV}}$ and $V_{\mathrm{PV}}$ denote the panel output current and voltage. $V_{\mathrm{PV} \text { cell }}$ denotes the PV cell voltage.

$I_{\text {sc }}$ denotes the short-circuit current under some insolation (denoted as $S$ ); we set the irradiance at standard test 


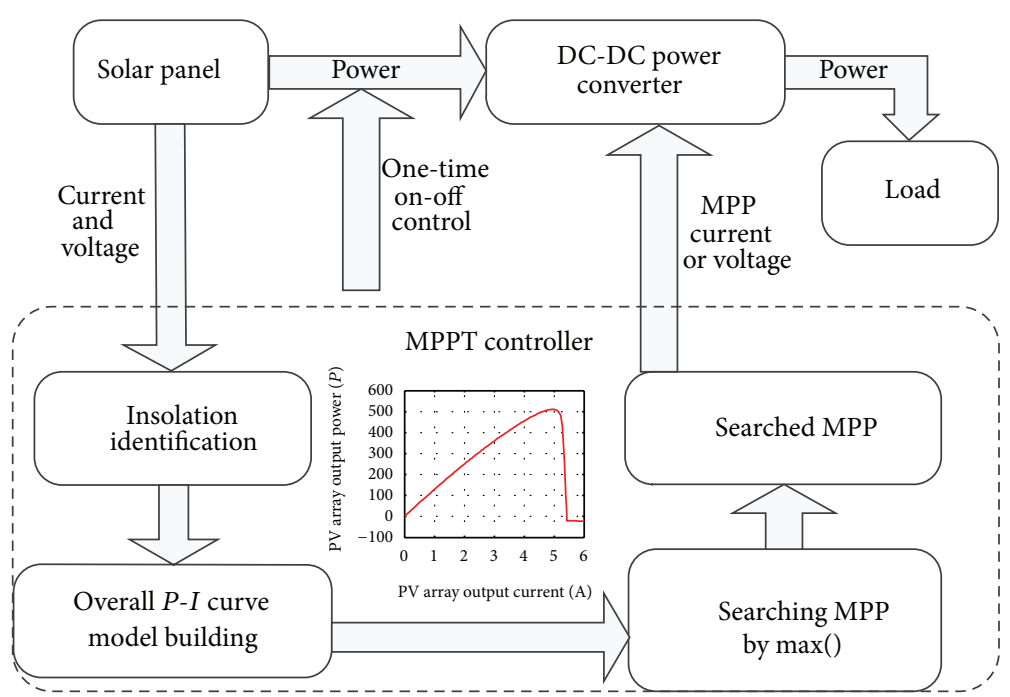

FIgURE 2: The proposed ultrafast MPP setting scheme.

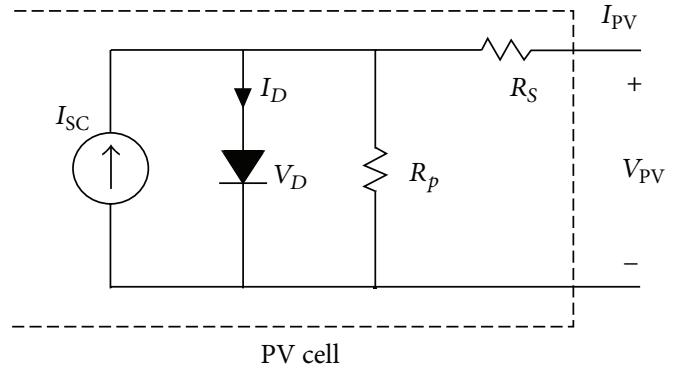

FIgURE 3: PV cell circuit model.

conditions (STC) as $I_{\text {sc,STC }}=1000 \mathrm{w} / \mathrm{m}^{2}$; the proportional gains from the insolation $I_{\mathrm{sc}, \mathrm{STC}}$ to the insolation $I_{\mathrm{sc}}$ are denoted as $G$; one derives $I_{\mathrm{sc}}=S * G=S * I_{\mathrm{sc}, \mathrm{STC}} / 1000$.

$I_{o}$ denotes the $\mathrm{PN}$-junction reverse saturation current. For the sake of convenience, the model parameters $I_{o}, R_{s}$, and $R_{p}$ are generally computed based on the model parameters (short-circuit current $I_{\mathrm{sc}}$, circuit voltage $V_{\mathrm{oc}}$, rated voltage $V_{r}$, and rated current $I_{r}$ ). The calculation of $I_{o}$ is denoted as

$$
I_{o}=\frac{\left(I_{\mathrm{sc}}-V_{\mathrm{oc}} / R_{p}\right)}{\left(\mathrm{e}^{V_{\mathrm{oc}} / V_{t}}-1\right)} .
$$

The current-input PV module model can be denoted as

$$
V_{\mathrm{PV}}=f\left(\text { ins, } I_{\mathrm{PV}}\right) \text {. }
$$

The voltage-input PV module model can be denoted as

$$
I_{\mathrm{PV}}=g\left(\text { ins, } V_{\mathrm{PV}}\right) \text {. }
$$

So, the model parameter identification problem can be expressed as

$$
\text { insolation }=\operatorname{ident}\left(\operatorname{dataset}\left(V_{\mathrm{PV}}, I_{\mathrm{PV}}\right)\right) .
$$

Based on sampling data, the model parameter identification procedure could be described as shown in Figure 4.

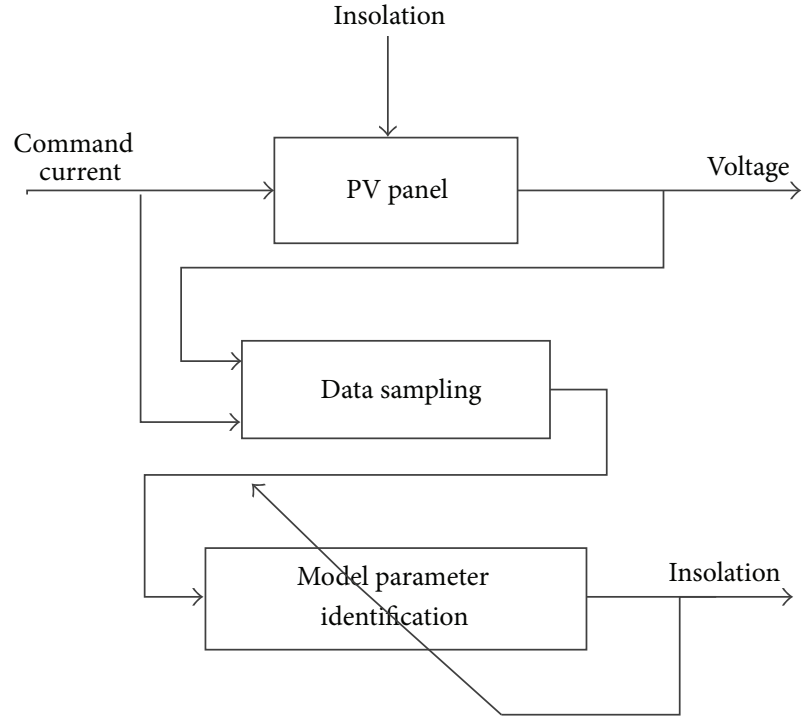

FIGURE 4: Flowchart for model parameter identification.

The current and voltage measured from the PV panel will be collected as a dataset sample for insolation parameter identification. Theoretically, only one sample dataset is needed for identifying the insolation if the current-input PV module model is known.

Furthermore, because the PV cell model is a nonlinear algebraic formulation without dynamics, the parameter identification problem can be actually defined as an algebraic equation solving problem. Therefore, the solving problem can be expressed as below:

$$
F(\text { ins })=V_{\mathrm{PV}}-f\left(\text { ins, } I_{\mathrm{PV}}\right)=0
$$

or

$G($ ins $)=I_{\mathrm{PV}}-g\left(\right.$ ins, $\left.V_{\mathrm{PV}}\right)=0$. 


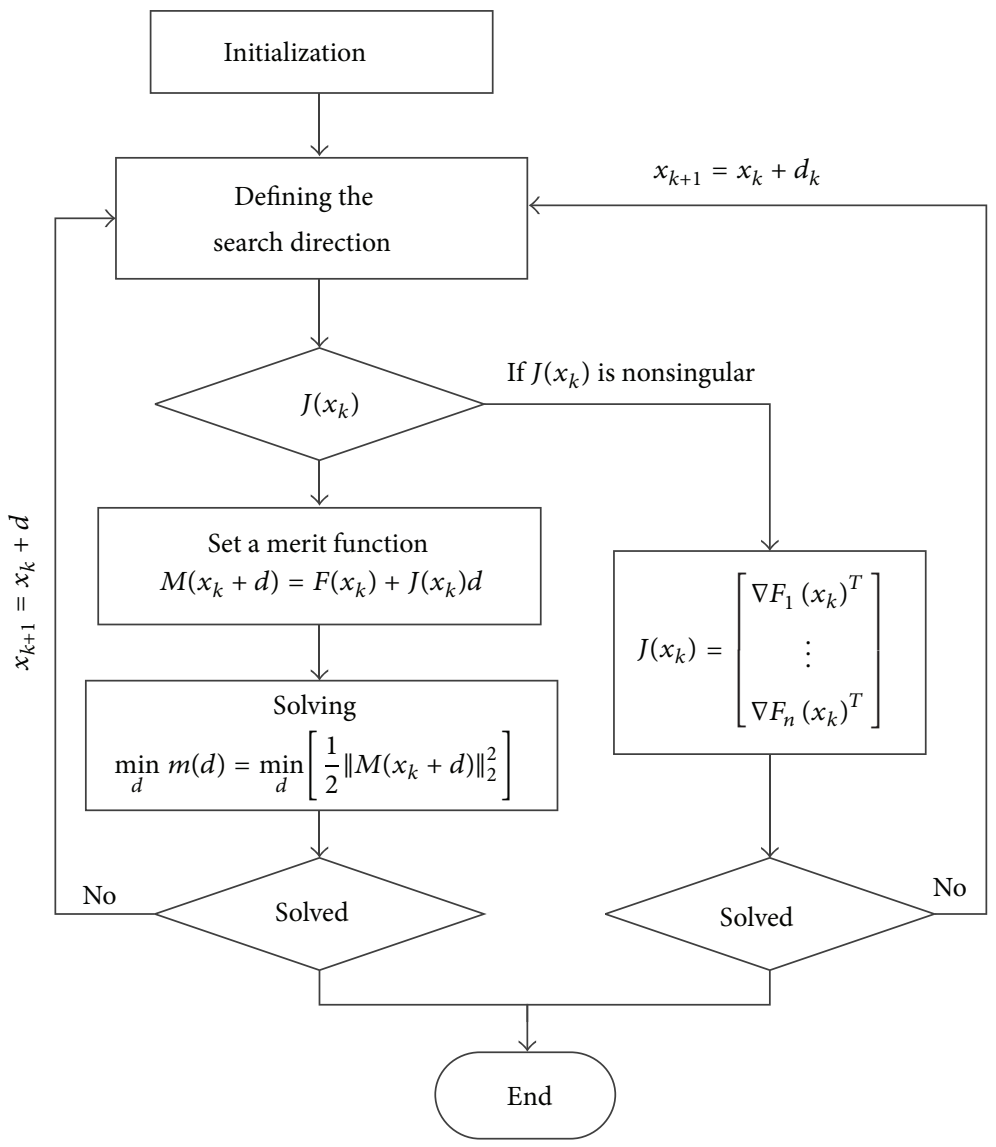

FIGURE 5: Flowchart for trust-region dogleg algorithm.

In order to solve this nonlinear algebraic equation to obtain the unknown variable ins, many of the methods used in optimization solvers are proposed. For the sake of generality and easy implementation for general microcomputers, a trust-region dogleg algorithm is employed in order to solve the PV equations with unknown insolation parameter.

The flowchart trust-region dogleg algorithm is presented in Figure 5. The key steps are defining the searching direction and process the nonsingularity of the Jordan matrix. The mathematical basis can be elaborated as below.

Given a nonlinear function set with $F_{i}(x)(i=1, \ldots, n)$, where $n$ is the dimensions of the vector $x$, the goal of equation set solving is to find a solution that satisfies all $F_{i}(x)=0$.

In order to obtain the solution, a Newton's method for linear system is firstly used to define the search direction. The search step $d_{k}$ is derived as below:

$$
\begin{gathered}
J\left(x_{k}\right) d_{k}+F\left(x_{k}\right)=0, \\
x_{k+1}=x_{k}+d_{k},
\end{gathered}
$$

where the matrix $J\left(x_{k}\right)$ satisfies:

$$
J\left(x_{k}\right)=\left[\begin{array}{c}
\nabla F_{1}\left(x_{k}\right)^{T} \\
\vdots \\
\nabla F_{n}\left(x_{k}\right)^{T}
\end{array}\right] .
$$

If $J\left(x_{k}\right)$ is nonsingular, step $d_{k}$ can be computed from (7). However, If $J\left(x_{k}\right)$ is singular, $d_{k}$ cannot be computed from (7) and a merit function is needed to address the singularity. To get the merit function, a function firstly is defined as follows:

$$
M\left(x_{k}+d\right)=F\left(x_{k}\right)+J\left(x_{k}\right) d .
$$

So, step $d_{k}$ is a root of (9), and it is also a root of a minimum value of the function $m(d)$, where

$$
\begin{aligned}
\operatorname{Min}_{d} m(d)=\operatorname{Min}_{d}\left[\frac{1}{2}\left\|M\left(x_{k}+d\right)\right\|_{2}^{2}\right] \\
=\operatorname{Min}_{d}\left[\frac{1}{2}\left\|F\left(x_{k}\right)+J\left(x_{k}\right) d\right\|_{2}^{2}\right] \\
=\operatorname{Min}_{d}\left[\frac{1}{2} F\left(x_{k}\right)^{T} F\left(x_{k}\right)+d^{T} J\left(x_{k}\right)^{T} F\left(x_{k}\right)\right. \\
\left.+d^{T} J\left(x_{k}\right)^{T} J\left(x_{k}\right) d\right]
\end{aligned}
$$

such that $\|D \cdot d\| \leq \Delta$.

So, the key problem here is to compute step $d$, which minimizes (10). 
By using a dogleg strategy, step $d$ can be chosen as below.

$$
d=d_{\mathrm{C}}+\lambda\left(d_{\mathrm{GN}}-d_{\mathrm{C}}\right)
$$

where $\lambda$ is the largest value in $[0,1]$ such that $\|d\| \leq \Delta$. And $d_{C}$ satisfy

$$
d_{C}=-\alpha J\left(x_{k}\right)^{T} F\left(x_{k}\right),
$$

where $\alpha$ is chosen to minimize (10) and $d_{\mathrm{GN}}$ satisfies

$$
J\left(x_{k}\right) d_{\mathrm{GN}}=-F\left(x_{k}\right) \text {. }
$$

As can be seen from the derivation above, the trust-region dogleg algorithm is efficient and easy to implement in microcomputers with numerical computation capability since it requires only one linear solving per iteration.

Comment 2. Because DSP numerical solvers are different from theoretical solving procedures, searching ranges for the trust-region algorithm need to be predefined. In our proposed scheme, this is generally known from the PV panels' characteristics. For example, the voltage range and power range are available from the specification of PV module while the irradiance is easy to measure or calculate.

3.2. Real-Time P-I Curve Generation. After the parameter insolation for each PV unit is solved from the PV cell equations, an overall $P-I$ or $P-V$ curve can be built based on the installation deployment of PV arrays. Generally, the PV panels are installed in series, so the PV panels are set in the same current and share the voltage drop. Assuming that the total unit number of PV panels or units with uniform $P-V$ characteristic is $n$, the overall PV module can be denoted as follows:

$$
V_{\mathrm{PV}}=\sum_{i=1}^{n} f\left(\mathrm{ins}_{i}, I_{\mathrm{PV}}\right) .
$$

And the overall output power can be denoted as

$$
P_{\mathrm{PV}}=I_{\mathrm{PV}} * \sum_{i=1}^{n} f\left(\mathrm{ins}_{i}, I_{\mathrm{PV}}\right) .
$$

In order to get a full picture of the overall $P-I$ curve model based on (15), a current input with a ramp format is defined to generate the full $P$-I curve model. The procedure of the $P-I$ curve model is shown in Figure 6. The current input is set as a ramp signal which covers the normal arrange of PV panel current. The overall power is calculated and then a $P-I$ curve model is generated.

3.3. Converter Operation with the MPP. Based on the P$I$ curve model constructed in the controller, a simple but effective algorithm function is used to search for the MPP of the curve. In our MPP searching scheme, the MATLAB function $\max (\cdot)$ is used to find the maximum point of the $P-I$ curve:

$$
[C, I]=\max (A),
$$

where it finds the indices of the maximum values of $A$ and returns them in output vector $I$. If there are several identical maximum values, the index of the first one found is returned. Because all of the algorithm operation is only executed in the controller and has no action with the converter, it is ultrafast and absolutely different from conventional MPPT algorithms. After the MPP is tracked in the controller, the converter will be executed at the MPP directly.

The converter operation in the complete MPPT process can be described as in Figure 7, with only two operations executed in a MPPT window observing period. The bluemarked part is executed by the MPPT controller. The execution time depends on the micro-computer performance and is very short because only numerical calculations are done. So, after the first converter operation, the converter will enter into the second-time operation very fast, and then it works at the MPP for a MPPT monitoring period.

\section{Simulation Results}

In order to validate the proposed MPP setting scheme, PV arrays with uniform insolation, nonuniform insolation, and time-varying insolation are tested. Corresponding results of three issues such as insolation parameter identification, generated real-time $P-I$ curves, and MPP tracking under insolation variation are presented here to show the effectiveness and superiority of the proposed method.

The PV panel parameters used in our work are listed below:

$$
\begin{gathered}
I_{\mathrm{sc}, \mathrm{STC}}=5.45 \mathrm{~A}, \\
V_{\mathrm{oc}}=22.2 \mathrm{~V}, \\
I_{P \max }=4.95 \mathrm{~A}, \\
V_{P \max }=17.2 \mathrm{~V}, \\
\operatorname{Gain}\left(\frac{\text { insolation }}{I_{\mathrm{sc}}}\right)=\frac{1}{1000} .
\end{gathered}
$$

4.1. Insolation Parameter Identification Result. In order to test the accuracy of parameter identification and simulate the measurement scenarios of the real converter controller, bandlimited white noise is injected into the measured signals to test the identification parameter's accuracy. A sine waveform variation of insolation, which varies from $495 \mathrm{w} / \mathrm{m}^{2}$ to $505 \mathrm{w} / \mathrm{m}^{2}$, is set for a PV panel model. The comparison result between real insolation and identification insolation is shown in Figure 8, and it can be concluded that the insolation identification parameter can track the real insolation at a high accuracy in the presence of measurement noise.

4.2. Generated Real-Time P-I Curves. In order to validate the effectiveness of real-time $P-I$ curve generation, a threepanel PV array model with nonuniform and time-varying insolation is simulated to test the proposed MPP setting 


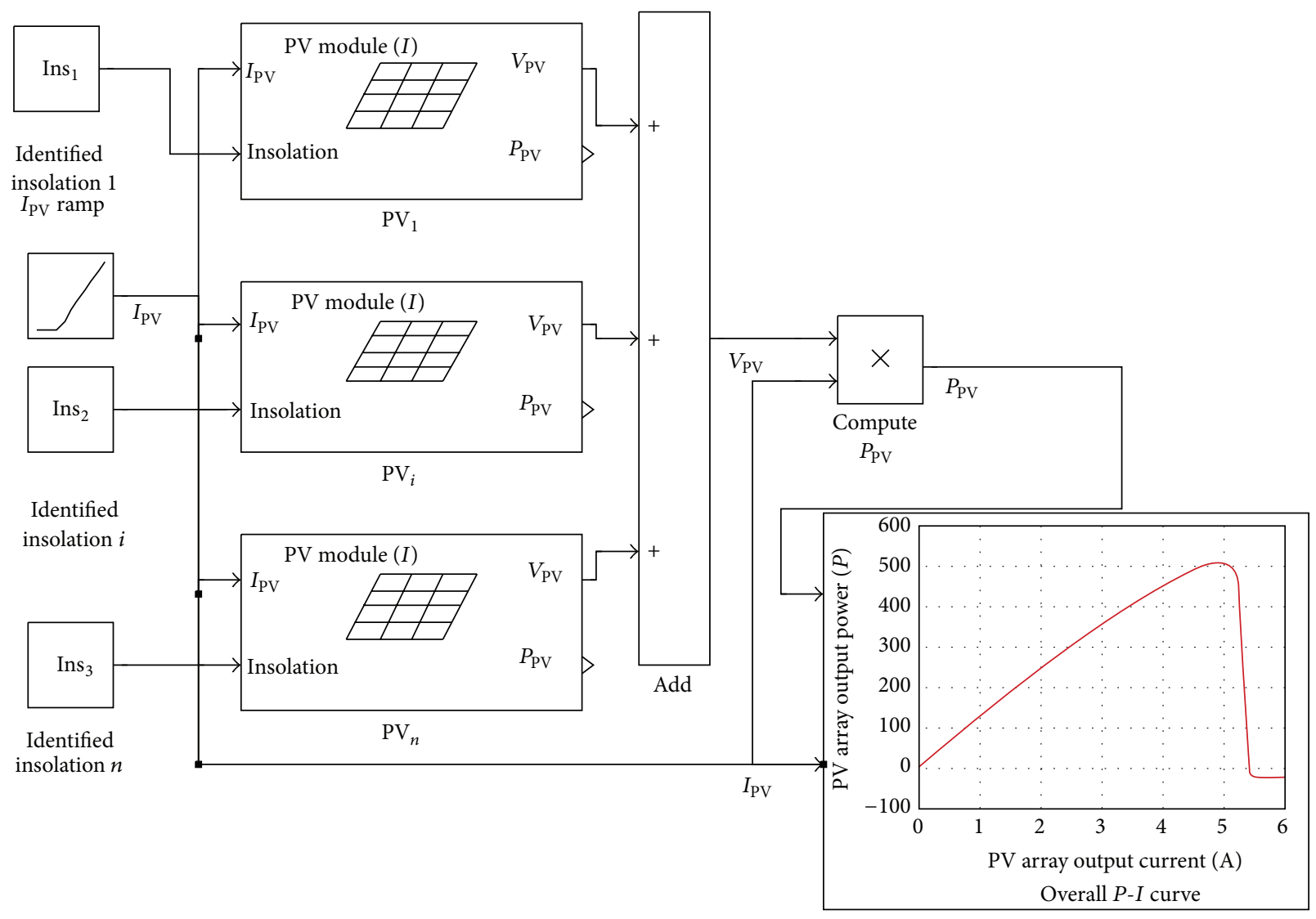

FIGURE 6: P-I curve model generated from ramp signal.

scheme. The insolation parameters of the three PV panels vary as below:

$$
\begin{aligned}
& \text { ins }_{1}=800 \mathrm{w} / \mathrm{m}^{2}, \\
& \text { ins }_{2}=800+100 \sin (2 \pi t) \mathrm{w} / \mathrm{m}^{2}, \\
& \text { ins }_{3}=900 \mathrm{w} / \mathrm{m}^{2} .
\end{aligned}
$$

Figure 9 depicts the real-time $P$ - $I$ curves generated by the proposed scheme. As can be seen from Figure 9, although the time interval is only $0.02 \mathrm{~s}$ and very short, the proposed scheme can still generate the $P-I$ curves at each instant in realtime. A dominant benefit of this fast response is that the MPP is available in a very short time interval in real-time and it will save a lot of time to avoid unnecessary energy losses when compared to conventional MPPT approaches.

\subsection{MPP Tracking under Different Insolation Conditions.} Figure 10 depicts the MPP tracking comparison under uniform and invariable insolation. Three MPP tracking approaches, P\&O, global MPPT algorithm in [19], and the proposed MPP setting scheme, are tested in the same insolation scenario. In order to guarantee the comparability of the three approaches, the MPPT operation time interval is set as $0.02 \mathrm{~s}$. Actually, subjected to response time delay of power converter switching, the time interval is generally set as $0.2 \mathrm{~s}$. The smaller the time interval, the higher the fastresponse requirement and cost of switching components. Here the MPPT operation time interval is set as $0.02 \mathrm{~s}$ in order to show the superiority of the proposed MPP setting scheme. As can be seen from the tracking trajectories in Figure 10, although all the three approaches can track the MPP eventually, the proposed scheme can track the MPP at the one-time interval $0.02 \mathrm{~s}$; the $\mathrm{P} \& \mathrm{O}$ can track the MPP at $1 \mathrm{~s}$ level, while the global MPPT algorithm can track the MPP at $1.2 \mathrm{~s}$. Consequently the proposed scheme has absolute advantage in MPP convergence and tracking. Additionally, the global MPPT algorithm needs more time because its search must cover the range of the $P-I$ curve.

Figure 11 depicts the same nonuniform and variable insolation as (18). Three MPP tracking approaches are also tested for comparison. As can be seen from Figure 11, the proposed MPP setting scheme also has the absolute advantage under nonuniform and variable insolation conditions; it can track the MPP closely with one-time interval $0.02 \mathrm{~s}$. The global MPPT can track at the points close to the MPP after $1 \mathrm{~s}$ and actually it will lose tracking if the insolation variation varies quickly at a large magnitude. The $\mathrm{P} \& \mathrm{O}$ will track the nonMPP peak points and become ineffective.

Based on the validation result presented above, it can be concluded that the proposed MPP scheming has significant advantages. 


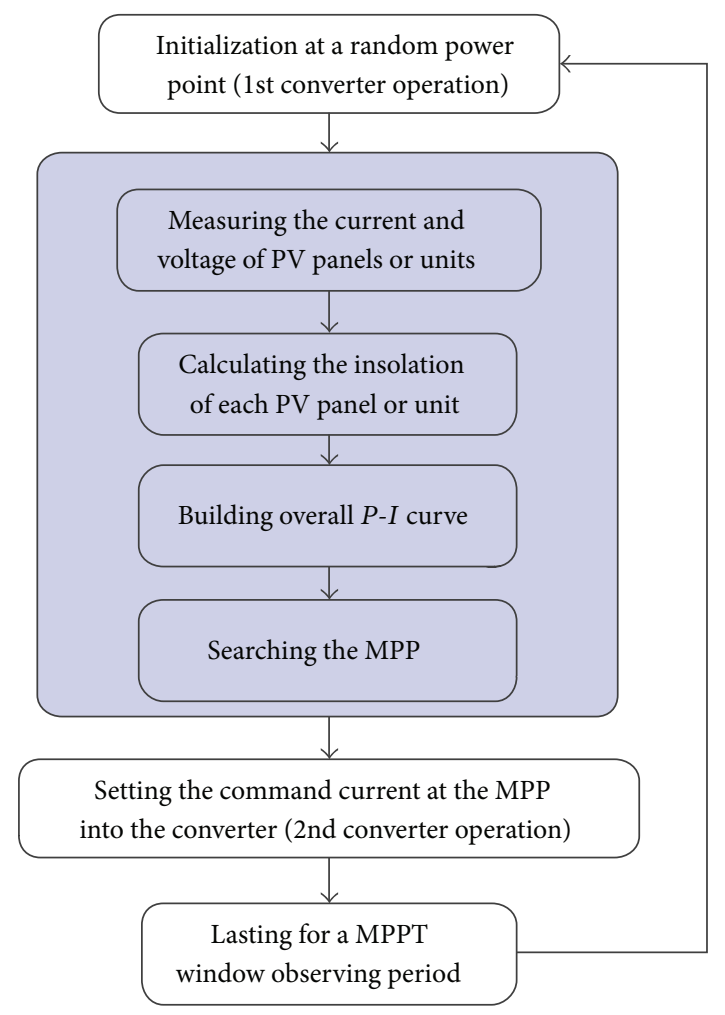

FIGURE 7: The proposed converter operation diagram.

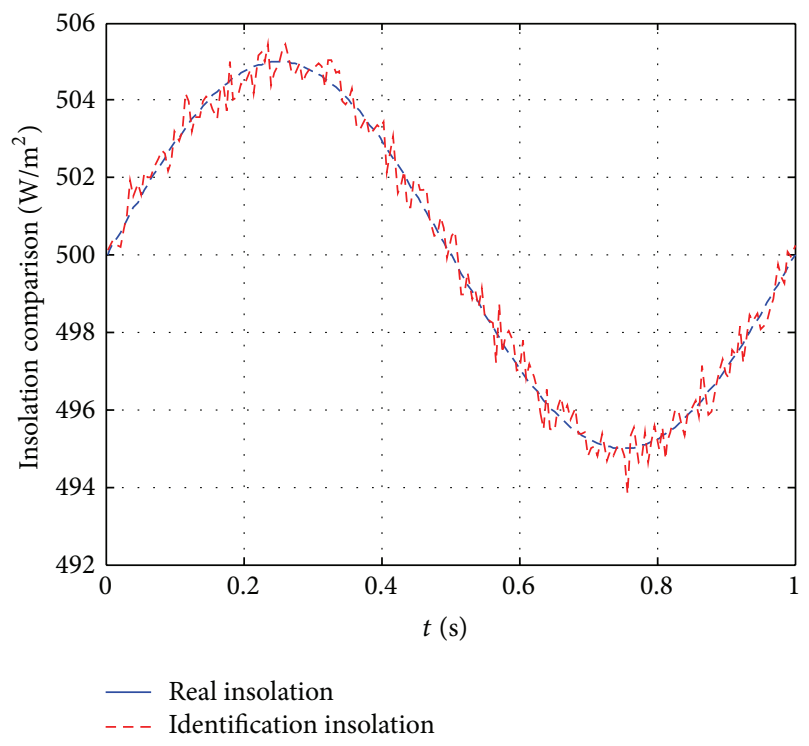

Figure 8: Comparison between real insolation and identification insolation.

(i) It can obtain the insolation parameter for each PV panel or unit but without the need to deploy additional insolation sensors, which is useful for monitoring large-scale solar arrays.

(ii) It only requires the power converter to perform two power operations during one MPPT tracking period.

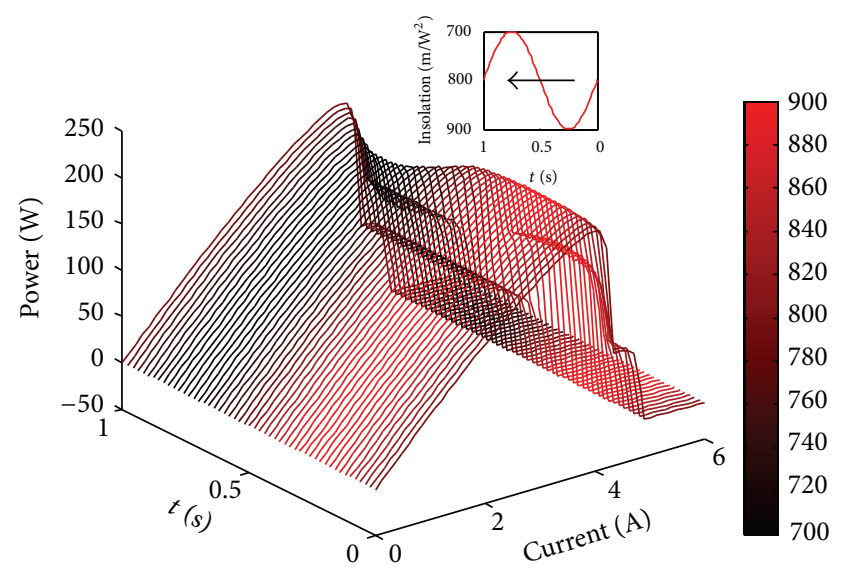

FIGURE 9: Generated real-time curves.

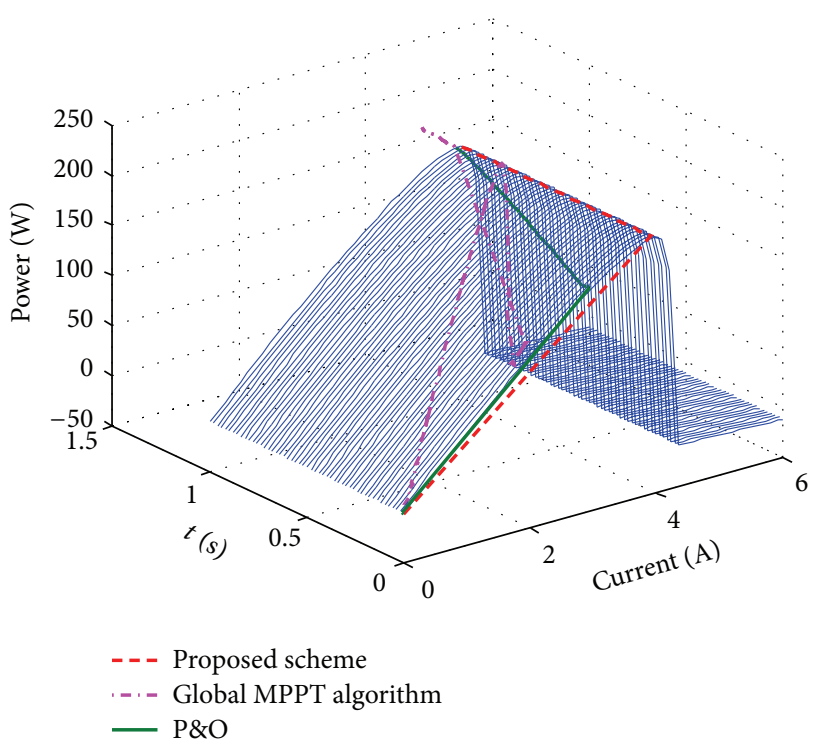

FIGURE 10: MPP tracking comparison under uniform and invariable insolation.

So it can reduce the unnecessary power losses in the conventional MPPT process.

(iii) It is ultrafast to monitor and track the MPP variation due to insolation changes. As a result, the proposed scheme has large potential to apply extreme adverse PV harvesting applications in the Middle East PV industry.

\section{Conclusions}

In order to improve the MPP tracking efficiency of PV harvesting, a novel MPP setting scheme based on model parameter identification is studied in this paper. Unlike previous MPPT methods, the proposed scheme can separate the MPP search from the power converter operation by identifying the key ambient condition parameter such as insolation and rebuilding the virtual $P-I$ curve in the controller. Consequently, the MPP tracking is ultrafast and 


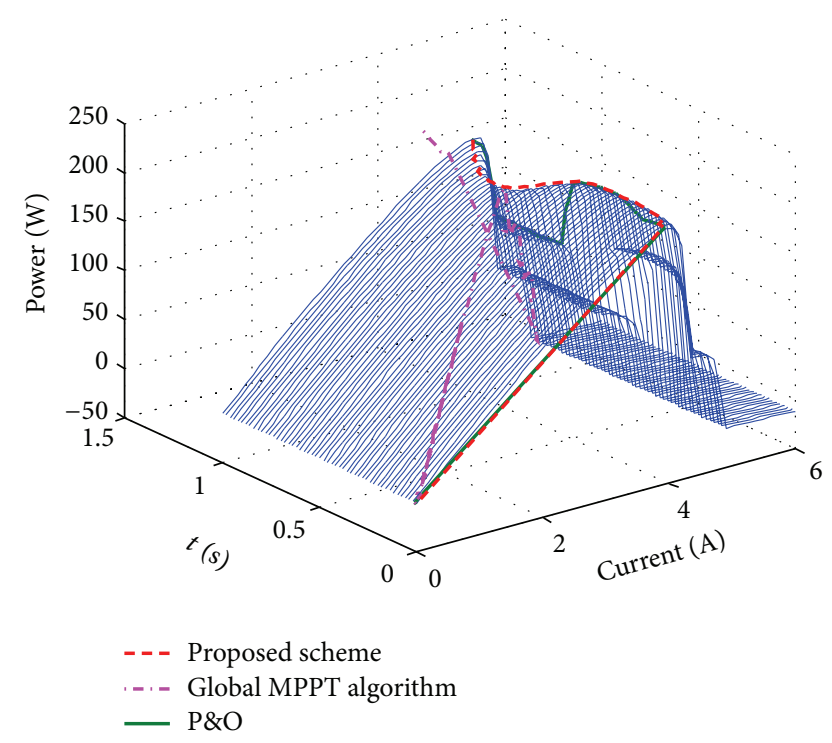

FIGURE 11: MPP tracking under nonuniform and variable insolation.

energy-saving because the frequent interaction between the controller and the converter is avoided. Finally, uniform, nonuniform, and time-varying insolation scenarios are simulated to validate its effectiveness and superiority. In this paper, the model identification approach is innovatively proposed to utilize useful information and reduce the conventional MPPT time and harvest more energy by calculating the $P-V$ curve in real-time and validate its feasibility and superiority in simulation. It is significantly superior to conventional methods; however, for some preconditions some flexibility is missing; it is a trade-off optimization which maximizes the PV power output by using some available information and resources. Our next work will focus on other cases with more unknown parameters and the experiential implementation for the proposed scheme.

\section{Conflict of Interests}

The author declares that there is no conflict of interests regarding the publication of this paper.

\section{Acknowledgments}

The author highly appreciates the reviewer's comments and Drs. Said A. Mansour and Antonio P. Sanfilippo from QEERI, Professor Paul Stewart at the University of Derby, UK, and Drs. Wenlong Ming and Yu Zeng at the University of Sheffield, UK, for their constructive comments on the research work.

\section{References}

[1] A. Bidram, A. Davoudi, and R. S. Balog, "Control and circuit techniques to mitigate partial shading effects in photovoltaic arrays," IEEE Journal of Photovoltaics, vol. 2, no. 4, pp. 532-546, 2012.
[2] F. Dinçer, "The analysis on photovoltaic electricity generation status, potential and policies of the leading countries in solar energy," Renewable and Sustainable Energy Reviews, vol. 15, no. 1, pp. 713-720, 2011.

[3] S. M. Moosavian, N. A. Rahim, J. Selvaraj, and K. H. Solangi, "Energy policy to promote photovoltaic generation," Renewable and Sustainable Energy Reviews, vol. 25, pp. 44-58, 2013.

[4] N. Onat, "Recent developments in maximum power point tracking technologies for photovoltaic systems," International Journal of Photoenergy, vol. 2010, Article ID 245316, 11 pages, 2010.

[5] K.-H. Chao, L.-Y. Chang, and H.-C. Liu, "Maximum power point tracking method based on modified particle swarm optimization for photovoltaic systems," International Journal of Photoenergy, vol. 2013, Article ID 583163, 6 pages, 2013.

[6] M. A. G. de Brito, L. Galotto, L. P. Sampaio, G. de Azevedo Melo, and C. A. Canesin, "Evaluation of the main MPPT techniques for photovoltaic applications," IEEE Transactions on Industrial Electronics, vol. 60, no. 3, pp. 1156-1167, 2013.

[7] R. Leyva, C. Olalla, H. Zazo et al., "MPPT based on sinusoidal extremum-seeking control in PV generation," International Journal of Photoenergy, vol. 2012, Article ID 672765, 7 pages, 2012.

[8] A. Mohamed and H. Shareef, "Hopfield neural network optimized fuzzy logic controller for maximum power point tracking in a photovoltaic system," International Journal of Photoenergy, vol. 2012, Article ID 798361, 13 pages, 2012.

[9] K.-H. Chao and Y.-H. Lee, "A maximum power point tracker with automatic step size tuning scheme for photovoltaic systems," International Journal of Photoenergy, vol. 2012, Article ID 176341, 10 pages, 2012.

[10] H.-T. Yau, C.-J. Lin, and C.-H. Wu, "Sliding mode extremum seeking control scheme based on PSO for maximum power point tracking in photovoltaic systems," International Journal of Photoenergy, vol. 2013, Article ID 527948, 10 pages, 2013.

[11] H. Mahamudul, M. Saad, and M. Ibrahim Henk, "Photovoltaic system modeling with fuzzy logic based maximum power point tracking algorithm," International Journal of Photoenergy, vol. 2013, Article ID 762946, 10 pages, 2013.

[12] R. Arulmurugan and N. Suthanthiravanitha, "Improved fractional order VSS Inc-Cond MPPT algorithm for photovoltaic scheme," International Journal of Photoenergy, vol. 2014, Article ID 128327, 10 pages, 2014.

[13] A. K. Abdelsalam, A. M. Massoud, S. Ahmed, and P. N. Enjeti, "High-performance adaptive Perturb and observe MPPT technique for photovoltaic-based microgrids," IEEE Transactions on Power Electronics, vol. 26, no. 4, pp. 1010-1021, 2011.

[14] R. Ramaprabha and B. L. Mathur, "A comprehensive review and analysis of solar photovoltaic array configurations under partial shaded conditions," International Journal of Photoenergy, vol. 2012, Article ID 120214, 16 pages, 2012.

[15] J. Shi, W. Zhang, Y. Zhang, F. Xue, and T. Yang, "MPPT for PV systems based on a dormant PSO algorithm," Electric Power Systems Research, vol. 123, pp. 100-107, 2015.

[16] C. Konstantopoulos and E. Koutroulis, "Global maximum power point tracking of flexible photovoltaic modules," IEEE Transactions on Power Electronics, vol. 29, no. 6, pp. 2817-2828, 2014.

[17] K. S. Tey and S. Mekhilef, "Modified incremental conductance algorithm for photovoltaic system under partial shading conditions and load variation," IEEE Transactions on Industrial Electronics, vol. 61, no. 10, pp. 5384-5392, 2014. 
[18] S. Palani, S. Peddapati, and K. Sundareswaran, "Application of random search method for maximum power point tracking in partially shaded photovoltaic systems," IET Renewable Power Generation, vol. 8, no. 6, pp. 670-678, 2014.

[19] A. Bousselham, Z. Cen, and A. Y. Elrayyah, "A global maximum power point tracking algorithm for photo-voltaic array with non-uniform insolation," in Proceedings of the 2nd International Renewable and Sustainable Energy Conference (IRSEC '14), pp. 548-553, Ouarzazate, Morocco, October 2014.

[20] K. Ding, X. Bian, H. Liu, and T. Peng, "A MATLAB-simulinkbased PV module model and its application under conditions of nonuniform irradiance," IEEE Transactions on Energy Conversion, vol. 27, no. 4, pp. 864-872, 2012.

[21] S.-H. Ko and R.-M. Chao, "Photovoltaic dynamic MPPT on a moving vehicle," Solar Energy, vol. 86, no. 6, pp. 1750-1760, 2012.

[22] F.-S. Pai, R.-M. Chao, S. H. Ko, and T.-S. Lee, "Performance evaluation of parabolic prediction to maximum power point tracking for PV array," IEEE Transactions on Sustainable Energy, vol. 2, no. 1, pp. 60-68, 2011.

[23] N. Adhikari, B. Singh, and A. L. Vyas, "Design, control and performance analysis of a standalone solar-PV energy generating system," International Journal of Renewable Energy Technology, vol. 6, no. 1, pp. 65-83, 2015.

[24] R. M. Chao, S. H. Ko, F. S. Pai, I. H. Lin, and C. C. Chang, "Evaluation of a photovoltaic energy mechatronics system with a built-in quadratic maximum power point tracking algorithm," Solar Energy, vol. 83, no. 12, pp. 2177-2185, 2009. 

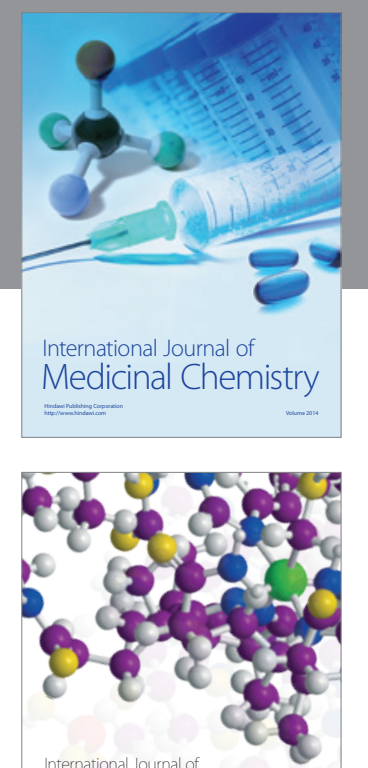

\section{Carbohydrate} Chemistry

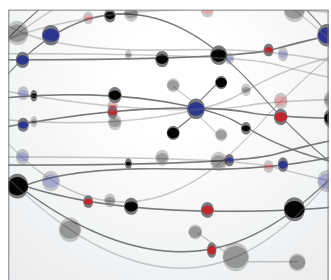

The Scientific World Journal
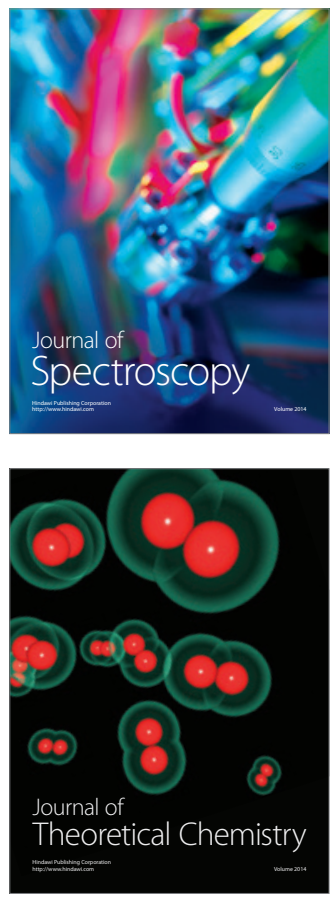
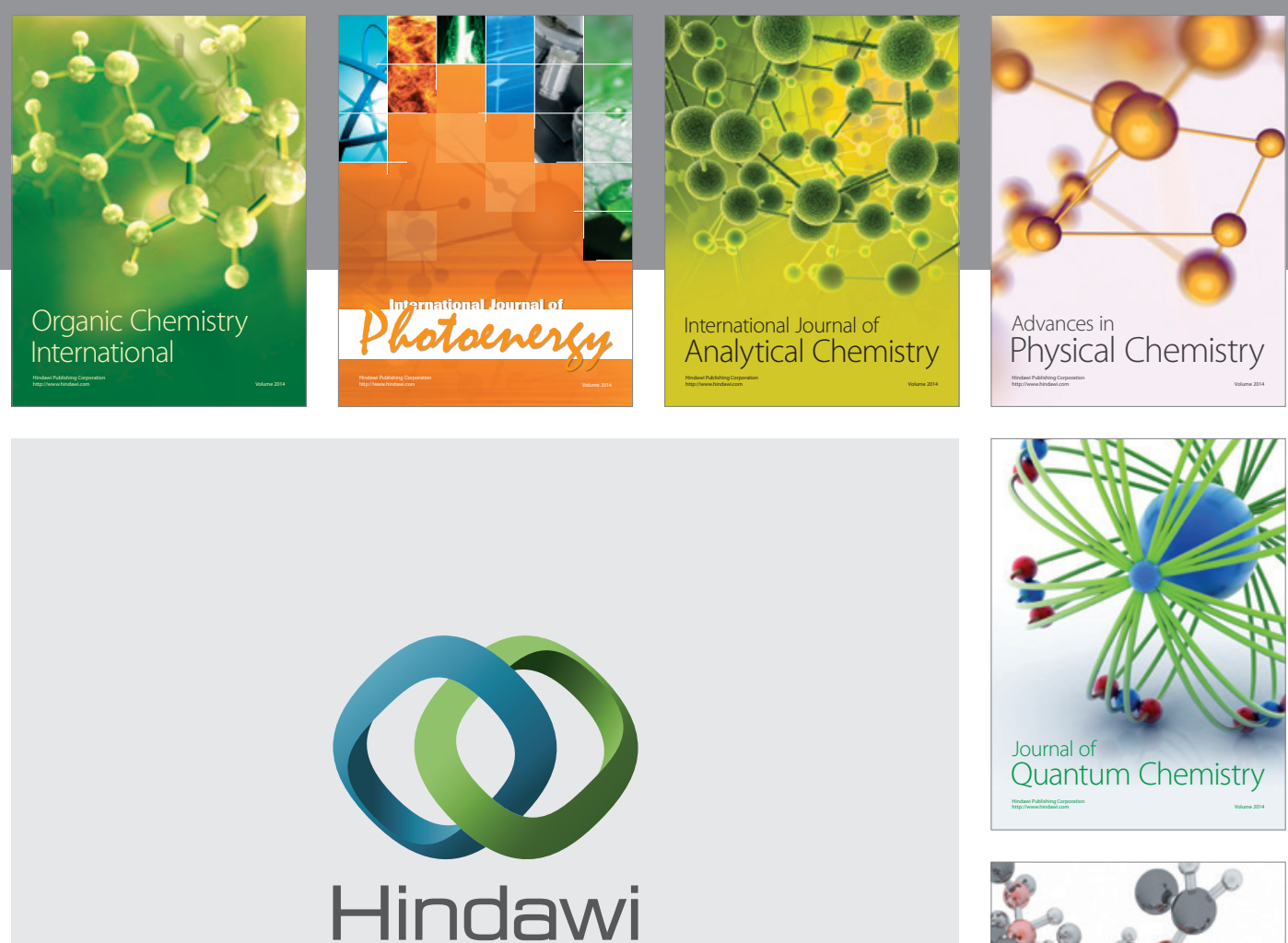

Submit your manuscripts at

http://www.hindawi.com

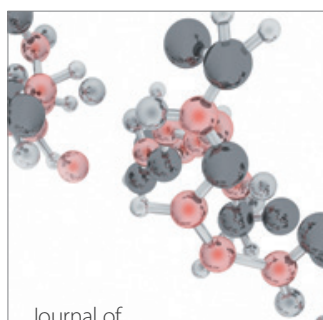

Analytical Methods

in Chemistry

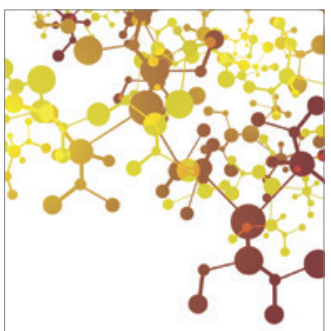

Journal of

Applied Chemistry

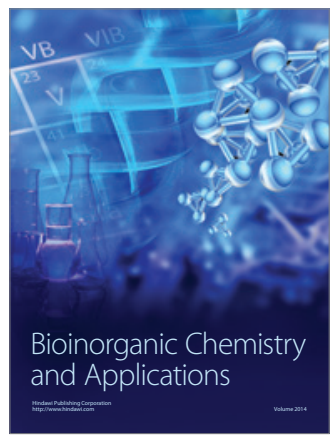

Inorganic Chemistry
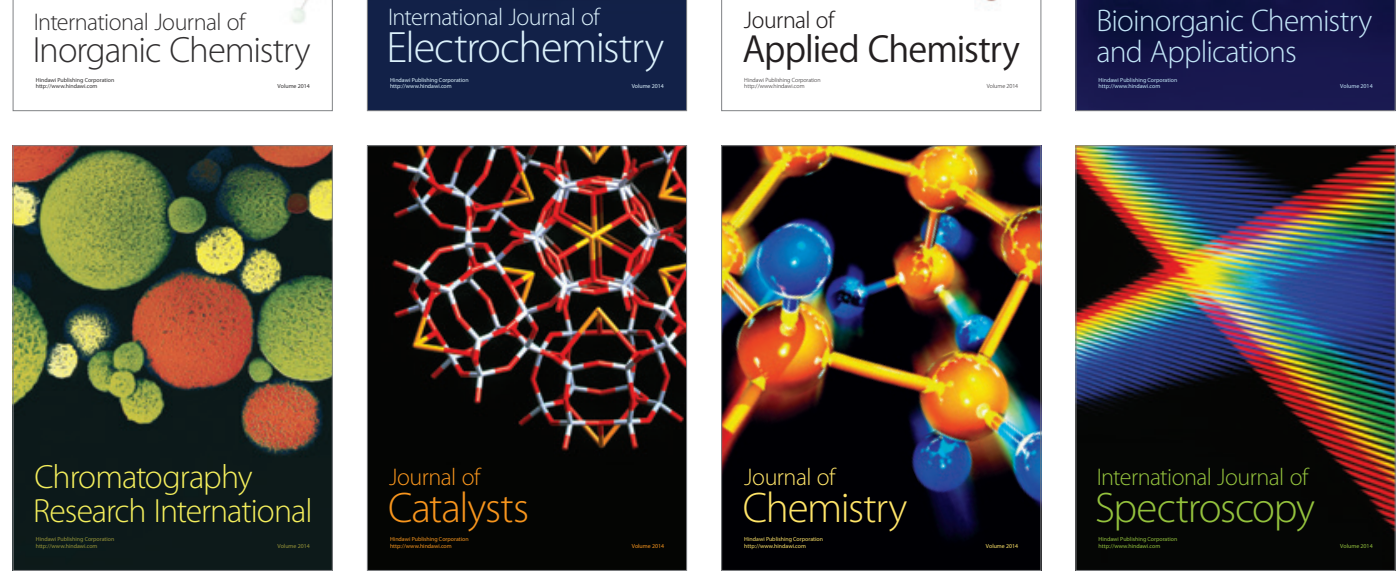